\section{Implementación del Primer Examen Clínico Objetivo Estructurado (ECOE) para la Certificación de la Especialidad en Anestesiología en Chile}

\author{
HÉCTOR J. LACASSIE ${ }^{1}$, MARCIA A. CORVETTO ${ }^{1}$, \\ ALEJANDRO E. DELFINO ${ }^{1}$
}

\section{Implementation of an Objective Structured Clinical Examination (OSCE) for Anesthesiology Certification in Chile}

\begin{abstract}
Anesthesiology became the first Chilean medical specialty certification board to incorporate an objective structured clinical examination (OSCE) into its certification system. The main reason for the introduction of an OSCE is to include an evaluation that allow candidates to demonstrate what they really "do" in clinical practice domains. Inherent in this justification is that the OSCE detects competences that are not well evaluated in current written and oral exams. This article describes the process of implementing an OSCE in Anesthesiology certification and a description of its application after one year of operation.
\end{abstract}

(Rev Med Chile 2020; 148: 1819-1824)

Key words: Anesthesiology; Certification; Clinical Competence.

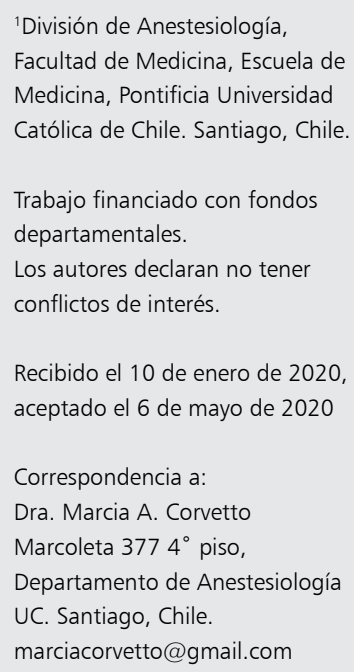

$\mathrm{D}$ e acuerdo a la legislación vigente en Chile, Especialidad es la rama de las ciencias de la salud cuyo objeto es una parte limitada de las mismas, sobre la cual poseen conocimientos, habilidades y destrezas definidas ${ }^{1}$. A su vez, se menciona que existen especialidades y subespecialidades, las que serán certificadas conforme a los requisitos establecidos en los programas de formación y en las normas técnicas operativas ${ }^{1}$. Las entidades certificadoras de especialidades que están acreditadas en Chile son: las universidades pertenecientes a la Asociación de Facultades de Medicina de Chile (ASOFAMECH); las universidades acreditadas por una agencia acreditadora ${ }^{2}$, y las agencias privadas acreditadas para dicha labor. Actualmente en Chile solo está acreditada la Corporación Nacional Autónoma de Certificación de Especialidades Médicas (CONACEM) como representante del último grupo ${ }^{3}$.

CONACEM fue fundada en 1984 y constituida por representantes de la Academia de Medicina del Instituto de Chile, Asociación de Facultades de Medicina de Chile, Colegio Médico de Chile A.G. y las Sociedades Científicas Médicas de Chile ${ }^{4}$. Desde los inicios de la Corporación, la especialidad de Anestesiología ha estado interesada en que se le reconozca como tal. De hecho, Anestesiología junto a ocho otras especialidades (cirugía, obstetricia, medicina interna, pediatría, traumatología y ortopedia, salud pública, medicina física y rehabilitación y urología), en 1987 fueron las primeras en acceder a la certificación de especialidad por CONACEM ${ }^{5}$.

El examen históricamente ha consistido en una parte teórica escrita, seguida de un examen práctico, realizado en algún centro certificado por CONACEM, en que hubiese disponibilidad para recibir a los participantes. En el año 2014 se estableció que la nota mínima aprobatoria del examen escrito era la nota cuatro $(4,0)$, lograda con 
$65 \%$ de respuestas correctas ${ }^{*}$. En esas condiciones, aquellos que aprobaban el examen accedían al examen práctico. Este último se realiza en un centro hospitalario, dependiente de una universidad, donde los evaluados no tienen privilegios clínicos, por tanto, solo se conversan casos, lo que termina siendo otro examen teórico, siendo imposible evaluar habilidades técnicas.

A contar de 2016, el influjo extranjero de especialistas aumentó exponencialmente, creciendo los postulantes a certificación en un factor de 10 veces en cuatro años ${ }^{5}$. Este aumento generó una rémora de postulantes para acceder al examen práctico que fue inmanejable con la estrategia empleada hasta esa fecha. El directorio de CONACEM decidió que aquellos postulantes que en el examen escrito obtuvieran un puntaje de $70 \%$ de respuestas correctas o más se eximirían del examen práctico y recibirán el certificado correspondiente ${ }^{* \star}$. Los postulantes que obtuviesen un resultado entre 65\% y $70 \%$ de correctas aún debían rendir el examen práctico. Si bien esta medida tiene validez por un año calendario (hasta marzo de 2020), el número de postulantes al examen práctico disminuyó significativamente. Aun así, la lista de espera era considerable, haciendo necesaria la instauración de alguna medida.

Dado que el examen práctico ha sido tradicionalmente el paso limitante para la certificación ya que las instituciones acreditadas para dicha labor son escasas, sumado a la gran heterogeneidad en su contenido y criterios de aplicación, se consideró que una forma de responder a estas falencias sería la creación de un Examen Clínico Objetivo Estructurado (ECOE) para la Certificación de la Especialidad en Anestesiología en Chile. Si bien esta metodología se ha aplicado en otras instancias de evaluación formativa en medicina ${ }^{6}$, su aplicación en certificación de especialidad es inédito en nuestro país y en Latinoamérica.

El examen está dirigido a postulantes a certificación de la especialidad por CONACEM. Son médicos (nacionales o extranjeros), que han cursado su especialización en una universidad o institución no acreditada en Chile, o bien han realizado su entrenamiento en práctica por 5 años en Chile;

\footnotetext{
*Instructivo CONACEM, 3 de octubre de 2014, Dr. José Manuel López, Presidente.

${ }^{* *}$ Carta a Presidentes de Comité, 14 de marzo de 2019, Dr. Lorenzo Naranjo T., Presidente.
}

que rindieron un examen escrito, lo aprobaron y ahora necesitan rendir el examen práctico.

Nuestro objetivo es describir la implementación del ECOE en Anestesiología para CONACEM y hacer una descripción de su aplicación al cabo de un año de funcionamiento.

\section{Método}

La División de Anestesiología y el Centro de Simulación de la Facultad de Medicina de la Pontificia Universidad Católica de Chile realizaron el examen práctico de la especialidad de Anestesiología para CONACEM. La metodología fue en base a un ECOE, evaluando competencias prácticas de la especialidad de Anestesiología. Los contenidos a evaluar fueron aquellos aspectos que todo anestesiólogo en Chile debiese saber, basados en los contenidos mínimos exigidos por CONACEM ${ }^{7}$. Además, se eligieron las competencias a evaluar por un grupo de expertos en simulación, para elegir el mejor tipo de simulación para evaluar cada una de las competencias elegidas.

Los temas a tratar fueron: manejo de vía aérea, reanimación cardiopulmonar, evaluación de pacientes en postoperatorio, manejo de drogas, evaluación preoperatoria en adultos y niños, técnicas anestésicas regionales, instalación de vías venosas centrales y periféricas, interpretación de exámenes preoperatorios, postoperatorios y manejo de complicaciones perioperatorias, entre otras.

La estructuración del examen fue con quince (15) estaciones orientadas a evaluar las competencias claves del quehacer anestesiológico, incluyendo estaciones de carácter teórico/práctico, práctico, en las cuales se utilizaron diferentes tipos de simulación según la competencia a evaluar, como simuladores de baja, mediana y alta fidelidad, así como de actores especialmente entrenados.

El esquema de contenido original se modificó a través de pruebas de concepto de escenarios utilizando pacientes o escenarios estandarizados. Este proceso iterativo equilibró el deseo de evaluar comportamientos relevantes, la necesidad de poder evaluar el desempeño de los candidatos de manera justa y la viabilidad de evaluar un gran volumen de candidatos dentro de un plazo razonable.

El examen consta de catorce o quince cubículos de examen (estaciones) en un circuito. Los candi- 
datos deben completar cada estación en sucesión. A su llegada deben leer la información relevante publicada fuera de cada cubículo. En ellas se les puede solicitar una demostración técnica o la interacción con un paciente, ya sea un maniquí de alta fidelidad o interpretado por un actor/ actriz. También hay estaciones donde el postulante responde preguntas escritas, habitualmente sobre interpretación de exámenes o situaciones clínicas. Estas estaciones fueron principalmente la interpretación de exámenes de laboratorio, rayos y electrocardiograma, que frecuentemente puede verse enfrentado un especialista. En general, las estaciones reflejan áreas relevantes de evaluación, entre las que se encuentran: 1) Reanimación cardiopulmonar; 2) Manejo de situaciones críticas en el quirófano; 3) Técnica de anestesia regional y accesos venosos; 4) Evaluación preoperatoria de adultos y niños; 5) Manejo de dolor agudo y crónico; 6) Interpretación de exámenes de laboratorio y situaciones clínicas.

Como pacientes estandarizados se utilizaron actores profesionales, especializados en escenarios y guiones biomédicos que fueron preparados con una a dos semanas de anticipación para permitir un tiempo suficiente para la revisión y el ensayo. Los guiones utilizados especifican las características físicas, el historial médico, la emocionalidad esperada del paciente, la información de fondo del escenario, las respuestas aceptables e inaceptables y las frases para estimular o cerrar una conversación.

Antes de la primera sesión, se realizó un ensayo general utilizando anestesiólogos certificados que se ofrecieron como candidatos de prueba. Esto permitió ajustar las estaciones y depurar detalles que de otra manera no hubiesen podido ser detectados.

El día que asistieron los participantes, previo al inicio del examen se tomó un tiempo extendido para explicar la metodología a usar y agenda de la sesión, utilizando elementos para establecer un contenedor seguro. Este concepto implica la adopción de conductas que hacen que exista un contexto sicológicamente seguro. Entre ellas se incluyen: 1) aclarar expectativas; 2) establecer un "contrato de ficción» con los participantes; 3) explicar los detalles logísticos y 4) declarar y promulgar un compromiso de respeto con los estudiantes y la preocupación por su seguridad psicológica? ${ }^{9}$. El tiempo requerido para la realización del examen fue de dos horas cronológicas, distribuidas en ocho minutos por estación, incluyendo el tiempo de recambio entre ellas. No se consideró estación de descanso. Las evaluaciones fueron realizadas por docentes dedicados a cada estación, contratados por el Centro de Simulación y por el Departamento de Anestesiología de la Universidad. Bajo estas condiciones, se aceptaron hasta catorce postulantes por evento, número concordante con otros exámenes de similares características y realidades ${ }^{8}$.

La evaluación del desempeño del examinado en cada escenario se basó en una lista de verificación que comprende 6-12 ítems. Esta fue desarrollada por un grupo de expertos a los que se les hizo una encuesta de consenso para llegar a un número mínimo de aspectos a evaluar. Luego, fue probada por voluntarios certificados en anestesiología quienes dieron sus opiniones para ajustar la pauta de evaluación antes de aplicarla en un examen formal. La lista de verificación se desarrolló de acuerdo a un formato rígido, sobre la base de "hecho"/"no hecho" y algunas estaciones incluyeron criterios necesarios de estar para aprobar la estación. La lista de verificación incluía tareas y secuencia de rendimiento. Todas las acciones incluidas en la lista de verificación fueron ponderadas por igual.

Durante el examen, un examinador completó la lista de verificación y el comité de examen recolectó los datos para su posterior análisis. Los examinados recibieron un puntaje de "aprobación" en el escenario si realizaron con éxito el 70\% de los elementos de la lista de verificación de la estación. Si la estación incluía algún criterio que debía estar presente para darse por aprobada, esta debía estar incluida en el 70\%. Las habilidades no técnicas evaluadas no fueron criterio reprobatorio de no estar presentes.

Una vez finalizado el examen, a los participantes se les proporcionó una encuesta a ser llenada anónimamente, en la que se indagó sobre sus percepciones en cuanto a ansiedad previa al examen, atingencia de las estaciones, preferencia de un examen de estas características sobre otras modalidades (examen oral tradicional), comodidad de las instalaciones y satisfacción en general con el examen, con puntaje de 0 a 10 . Luego de la primera fecha realizada, se desarrolló un video informativo, que se envió a los postulantes de la siguiente fecha, una semana antes de su examen. Se indagó en una siguiente versión de la encuesta si esta intervención tuvo un impacto en la percepción general del examen. 
A su vez, los docentes proporcionaron comentarios sobre el rendimiento de su estación y sobre posibles contratiempos o situaciones destacables durante el examen (debriefing).

\section{Resultados}

En el primer año de aplicación del ECOE para CONACEM se realizaron cuatro exámenes que incluyeron a 54 participantes, donde $33(61 \%)$ eran de sexo femenino y 21 de sexo masculino. Sus nacionalidades fueron 44 venezolanas $(81 \%)$, 6 cubanas $(11 \%)$ y 4 de otras nacionalidades $(8 \%)$.

La tasa de aprobación global del examen fue de $76 \%$, sin existir diferencias en los resultados al analizarlas por sexo o nacionalidad (test de Fisher, valor $\mathrm{p}=1$ ) o nacionalidad.

Las tasas de aprobación en las estaciones de reanimación cardiopulmonar para los 4 períodos de evaluación fueron 12/14 (86\%), 13/14 (93\%), $10 / 12(83 \%)$ y $11 / 14(79 \%)$. Las tasas de aprobación para la estación de manejo de manejo de crisis en el quirófano a fueron 35/42 (83\%), 34/42 (81\%), 34/36 (94\%) y $25 / 28$ (89\%). Aquellas para el manejo de técnica de anestesia regional y accesos venosos fueron 26/28 (93\%), 25/28 (89\%), $18 / 24(75 \%)$ y $25 / 28$ (89\%). Para la evaluación preoperatoria de adultos y niños las cifras fueron: $18 / 28$ (64\%), $27 / 42$ (64\%), $28 / 36(78 \%)$ y $36 / 42$ $(86 \%)$, mientras que para manejo de dolor agudo, crónico e interpretación de exámenes de laboratorio y situaciones clínicas fueron 11/14 (79\%), $13 / 14$ (93\%), $2 / 12$ (17\%), $14 / 14(100 \%)$ y $29 / 56$ (52\%), 32/56 (57\%), 26/48 (54\%) y 20/56 (36\%), respectivamente. Al analizar la tasa de respuestas correctas por cada dimensión estudiada, la tasa de resultados para la interpretación de exámenes de laboratorio y situaciones clínicas fue significativamente menor que para las otras áreas (Test de $\chi^{2}$, valor $\left.\mathrm{p}<0,001\right)$.

Los postulantes contestaron la encuesta, donde comentaron que el examen es muy atingente y las estaciones adecuadas, de acuerdo a los comentarios en texto libre de aquel acápite de la encuesta. Además, fueron concordantes en indicar que esta modalidad la prefieren por sobre la forma tradicional y que repetirían la experiencia si llegara el caso. Con respecto a la percepción de ansiedad previa al examen, tuvieron una mediana (rango intercuartil): 9,5 (8-10), mientras que post examen fue de $5(3,25-7,75)$ : valor $\mathrm{p}=0,007$ (Mann
Whitney U test). A su vez, mencionaron que la percepción de ansiedad para otro examen de similares características es de $5(3,25-7,75)$ (valor $\mathrm{p}=$ no significativo $)$.

En las siguientes fechas, todos los participantes recibieron un link con un video informativo, que revisaron previo al examen. La nueva encuesta destacó el análisis de ansiedad previa al examen con una mediana (rango intercuartil): 9 (8-10) y post examen 4 (3-5): valor $\mathrm{p}=0,0001$ (Mann Whitney $U$ test). A su vez, mencionaron que la percepción de ansiedad para otro examen de similares características sería de $5(3,0-6,75)$. Aparentemente el video contribuyó a disminuir la ansiedad pre examen (valor $\mathrm{p}=0,0026$ (Mann Whitney U test)), aunque probablemente no es el único factor que influya.

\section{Discusión}

La metodología utilizando un ECOE complementando la evaluación de especialidad médica, en particular en anestesiología, se ha empleado hace más de diez años en Reino Unido e Israel ${ }^{10,11}$. En Estados Unidos de Norteamérica, el ECOE se agregó al proceso de certificación de anestesiología en 2018. A su vez, la Junta Americana de Anestesiología fue el primer ente de certificación de especialidad médica de los Estados Unidos en incorporar este tipo de evaluación en su sistema de examen de certificación ${ }^{8}$, situación homóloga a lo que ocurrió solo unos meses después en Chile. La incorporación de un ECOE fue la evolución lógica a una necesidad en cuanto a calidad como a cantidad de los exámenes a ser aplicados.

La razón fundamental para la introducción del ECOE es aprovechar la fidelidad, la estandarización y la reproducibilidad que proporciona esta metodología para permitir que los candidatos se desempeñen en los dominios probados. Inherente a esta lógica es que el ECOE captura competencias importantes para la práctica de la anestesiología que no se evalúan bien en los exámenes escritos y orales actuales ${ }^{8}$. Por ejemplo, con este tipo de evaluación somos capaces de decir si el anestesiólogo es capaz de manejar un paro cardiorrespiratorio o si es capaz o no de instalar un catéter venoso central. Adicionalmente este examen nos permite evaluar distintos tipos de habilidades. Tiene la capacidad de evaluar habilidades cognitivas y motoras en procedimientos, junto con habilidades no técnicas ${ }^{12}$. 
El examen que actualmente se aplica ha demostrado en forma consistente en las diferentes fechas de aplicación, que los participantes adolecen de ciertos conocimientos propios del quehacer anestesiológico, en especial en cuanto a la interpretación de exámenes de laboratorio y situaciones clínicas. Si bien los participantes podrían desempeñarse razonablemente bien en la mayoría de las situaciones evaluadas en el examen, esta brecha de conocimiento pudiera generar morbilidad en los pacientes, lo que es ciertamente preocupante. Las estaciones más conflictivas en términos de aprobación por los postulantes fueron analizadas y probadas por diferentes anestesiólogos de variada experiencia, validándolas previo a su aplicación y probándolas en su desempeño, por lo que es improbable que el mal desempeño se deba a una complejidad excesiva.

El fenómeno migratorio especialmente desde Venezuela ha obligado a buscar alternativas para resolver una lista de espera en el proceso de certificación de especialidad en Chile ${ }^{13}$. La incorporación de esta modalidad de selección logró reducir considerablemente los tiempos de proceso ni las tasas históricas de aprobación, reportándose en el período 20022015: Examen escrito aprobado: 129/242 (53,3\%); Examen práctico aprobado: $177 / 195(90,8 \%)^{14}$. En esta ocasión, la tasa de aprobación fue de $76 \%$, sugiriendo que el ECOE tiene capacidad discriminatoria homologable o mejor a la modalidad original. A su vez, esta nueva modalidad ha reducido considerablemente el tiempo requerido para examinar a los postulantes. Con el examen tradicional, hubiese tomado al menos un año, asumiendo un examen semanal y que los centros examinadores aceptaran postulantes durante todo el año.

Cabe mencionar que el examen escrito realizado bianualmente por CONACEM sigue siendo el principal filtro en el proceso de certificación de la especialidad. La tasa de aprobación desde 2014 a 2018 fue de $47,1 \%$, con una calificación promedio de 62,9\% (IC 95\% 61,7\%-64,1\%). De ese universo, de acuerdo a las directrices actuales, solo rendirá el examen práctico $14,8 \%$ de los postulantes y $31,4 \%$ de quienes aprueban el examen escrito. Eso implica que los postulantes al ECOE son entre 20 a 30 candidatos anuales, cifra perfectamente manejable por este sistema, que es complementario al tradicional, que aún sigue en práctica. El gran aporte de la incorporación del ECOE fue reducir en un corto plazo la lista de espera de postulantes que estaban en situación de necesidad de dar su examen práctico como último requisito para la certificación. En este caso, considerando el período entre 2014 y 2018, eran 56 médicos.

Luego de la primera fecha realizada, se desarrolló un video informativo, que se envió a los postulantes de la siguiente fecha, una semana antes de su examen. Se indagó en una siguiente versión de la encuesta si esta intervención tuvo un impacto en la percepción general del examen. Aparentemente habría tenido un efecto positivo en disminuir la ansiedad anticipatoria al examen, permitiendo que los participantes se concentraran en el examen propiamente tal y no en aspectos adicionales que no debieran interferir en el desempeño real.

Las limitaciones del actual modelo son variadas y se ha intentado dar cuenta de ellas. Estamos conscientes que hay traspaso de información entre postulantes de diferentes fechas de examen. Si bien esta es una situación que siempre va a ocurrir y no es posible controlarla, los postulantes no conocen la pauta de evaluación y, por lo tanto, no basta con saber de qué se trata el escenario. El desempeño tiene una secuencia tal que, de no cumplirse, el postulante arriesga la no aprobación del mínimo requerido en la estación; el número mínimo de estaciones es de $14^{15,16}$, ya que bajo ese número no se alcanza a evaluar los contenidos mínimos y puede comprometer la especificidad del examen para detectar los postulantes más débiles y que no debieran aprobar. Con esto, se requiere un gran número de evaluadores. Al existir actores y actrices simulando pacientes, siempre hay vulnerabilidad en que no se presenten el día del examen, y dado que los personajes son específicos, es difícil el reemplazo a última hora.

El desafío futuro es continuar mejorando esta iniciativa, iterando cada una de las estaciones y mejorando las herramientas de evaluación. Posteriormente, lograr transferir esta experiencia a otras especialidades. En este momento ya se inició el examen para la especialidad de cirugía. Las directrices a futuro son incorporar otras especialidades como ginecología y pediatría.

Adicionalmente, creemos que este es el primer paso de estandarización de certificación de habilidades en anestesia, que puede dar pie al desarrollo de certificación y recertificación de especialidades en nuestro país para los programas nacionales. Incluso, algún día podría llegar a una estandarización de certificación latinoamericana. 
Sería relevante que otros centros desarrollen la capacidad de implementar esta modalidad de examen a fin de aumentar la capacidad examinadora, además de disminuir el desarrollo de potenciales sesgos que pudieran aparecer por ser solo una institución la que está a cargo de esta modalidad de examen.

En resumen, presentamos la implementación del ECOE en Anestesiología para CONACEM y la descripción de sus resultados luego del primer año de funcionamiento. Ha demostrado ser una herramienta funcional y homologable al examen tradicional en cuanto a resultados, pero con una adición de objetividad y reproducibilidad que exige una evaluación tan relevante como esta. Es deseable que se masifique a otras especialidades y a otras instancias como la recertificación de especialidades.

\section{Referencias}

1. Ministerio de Salud. DS $\mathrm{N}^{\circ}$ 8: REGLAMENTO DE CERTIFICACIÓN DE LAS ESPECIALIDADES DE LOS PRESTADORES INDIVIDUALES DE SALUD Y DE LAS ENTIDADES QUE LAS OTORGAN. Type of Medium. Fecha de última actualización: Last Update Date; Contents Disponible en: http://www.supersalud. gob.cl/normativa/668/articles-8925_recurso_1.pdf

2. Chacon R. Formación de anestesistas en Chile: cómo ha evolucionado la formación de nuestra especialidad en los últimos 40 años. Rev Chil Anest 2014; 43: 165-88.

3. Ministerio de Salud. RESOLUCIÓN EXENTA N ${ }^{\circ} 399$. AUTORIZA A LA CORPORACIÓN NACIONAL AUTÓNOMA DE CERTIFICACIÓN DE ESPECIALIDADES MÉDICAS “CONACEM” COMO ENTIDAD CERTIFICADORA DE ESPECIALIDADES MÉDICAS QUE SE INDICAN. Type of Medium. Fecha de última actualización: Last Update Date; Contents Disponible en: https://www.minsal.cl/wp-content/uploads/2016/02/ Resoluci\%C3\%B3n-Autorizaci\%C3\%B3n-CONACEM-2014-02-17.pdf

4. Corporación Nacional Autónoma de Certificación de Especialidades Médicas. CONACEM. Fecha de última actualización: 7-12-2019; [Citado el 30 de diciembre de 2019]. Disponible en: http://www.conacem.cl/

5. Lacassie HJ. Certificación, recertificación y recontracertificación en anestesiología. Rev Chil Anest 2019; 48 (4): 286-7.

6. Triviño BX, Vásquez MA, Mena MA, López TA, Aldunate RM, Varas PM, et al. Aplicación del Examen Clínico Objetivo Estructurado (OSCE) en la evaluación final del internado de pediatría en dos escuelas de medicina. Rev Med Chile 2002; 130: 817-24.

7. Corporación Nacional Autónoma de Certificación de Especialidades Médicas. REQUISITOS POR ESPECIALIDAD PARA LA CERTIFICACIÓN DE ESPECIALISTAS. Fecha de última actualización: 7-12-2019; [Citado el 30-12-2019]. Disponible en: http://www.conacem.cl/ requisitos_especialidad.asp

8. Warner DO, Isaak RS, Peterson-Layne C, Lien CA, Sun $\mathrm{H}$, Menzies AO, et al. Development of an Objective Structured Clinical Examination as a Component of Assessment for Initial Board Certification in Anesthesiology. Anesth Analg 2020; 130 (1): 258-64.

9. Rudolph JW, Raemer DB, Simon R. Establishing a safe container for learning in simulation the role of the presimulation briefing, Simulation in Healthcare: Journal of the Society for Simulation in Healthcare 2014, p 339-49.

10. Berkenstadt H, Ziv A, Gafni N, Sidi A. Incorporating simulation-based objective structured clinical examination into the Israeli national board examination in anesthesiology. Anesth Analg 2006; 102 (3): 853-8.

11. Royal College of Anaesthetists. Primary FRCA OSCE. Fecha de última actualización: mayo 2018; [Citado el 30 de diciembre de 2019]. Disponible en: https://www. rcoa.ac.uk/examinations/primary-frca-examinations/ primary-frca-osce

12. Sidi A, Baslanti TO, Gravenstein N, Lampotang S. Simulation-based assessment to evaluate cognitive performance in an anesthesiology residency program. J Grad Med Educ 2014; 6 (1): 85-92.

13. Cabello N, Chávez M. Acuerdo de Conacem permitirá agilizar la certificación de profesionales: Casi 500 nuevos médicos especialistas podrán incorporarse y atender en hospitales públicos., El Mercurio, Santiago, 21 de marzo de 2019, Cuerpo C, C4. Disponible en: http://www. clinicasdechile.cl/noticias/casi-500-nuevos-medicos-especialistas-podran-incorporarse-y-atender-en-hospitales-publicos/

14. CONACEM. En: Edited by López Moreno, JM, Hervé Allamand, L. Capítulo 7: Certificación de Especialistas, Corporación Nacional Autónoma de Certificación de Especialidades Médicas (1984-2015). Santiago: Gráfica LOM, 2016, p. 65-174.

15. Jalili M, Hejri SM, Norcini JJ. Comparison of two methods of standard setting: the performance of the three-level Angoff method. Med Educ. 2011; 45 (12): 1199-208.

16. Trivino X, Vásquez A, Mena A, López A, Aldunate M, Varas M, et al. [Application of Objective Structured Clinical Examination (OSCE) for pediatric internship assessment in two schools of medicine]. Rev Med Chile 2002; 130 (7): 817-24. 